\title{
A Tale of Two Provinces: Regionalization of Pancreatic Surgery in Ontario and Quebec
}

\author{
Christopher J. Sonnenday, MD, MHS and John D. Birkmeyer, MD \\ Michigan Surgical Collaborative for Outcomes Research and Evaluation (MSCORE), The University of Michigan, \\ Ann Arbor, MI
}

Evidence has accumulated over several decades suggesting that high-volume surgeons and hospitals achieve better clinical results with selected surgical procedures. ${ }^{1,2}$ Volume-outcome relationships are particularly strong for high-risk but infrequently performed procedures such as esophagectomy, hepatic resection, and pancreatic resection, where low-volume providers may perform only a few operations a year. In the United States, efforts to concentrate such procedures in high-volume centers have included selective referral initiatives by payer coalitions, public reporting of hospital caseloads by Internet rating firms, and guidelines from professional organizations. ${ }^{3}$ However, it remains unclear whether these efforts have been successful in concentrating care or, more importantly, in reducing mortality with high-risk procedures.

In this issue of Annals of Surgical Oncology, Simunovic and colleagues describe the effects of an explicit attempt by Canadian authorities to regionalize one procedure: pancreatic resection. ${ }^{4}$ Beginning in 1999, Cancer Care Ontario initiated a series of quality improvement initiatives aimed at limiting that procedure to hospitals performing $\geq 10$ procedures a year, with an operative mortality of $<5 \%$. This initiative was remarkably successful in concentrating care (reflected both by increasing median hospital volumes and declining numbers of hospitals performing pancreatic resection) and even more effective in reducing mortality. Overall, operative mortality in Ontario decreased from $10.4 \%$ in 1994 to less than $2.2 \%$ in 2004 . Simunovic et al. also present paradoxical findings from the province of Quebec, which had made no similar attempt to regionalize or otherwise improve care with that procedure. ${ }^{4}$

(C) Society of Surgical Oncology 2010

Published Online: 25 May 2010

C. J. Sonnenday, MD, MHS

e-mail: csonnend@umich.edu
Over the same decade, pancreatic resection became increasingly concentrated in high-volume centers, though to a slightly lesser degree than in Ontario. However, there were no commensurate reductions in operative mortality. In 2004 , overall mortality remained at almost $10 \%$.

The experience in Ontario represents some of the best evidence to date of the power of volume-based regionalization. Operative mortality declined occurred virtually overnight-from $8.7 \%$ in 1999 to $3.3 \%$ in 2000. It is difficult to attribute this improvement to chance or any other factor. Mortality continued to decline subsequently with increasing hospital caseloads, further evidence of the causal relationship between the regionalization initiative and patient outcomes. Emerging data from other countries also support the value of regionalization in reducing mortality with highrisk cancer procedures. In our unpublished analyses of the United States Medicare population, operative mortality with both pancreatectomy and esophagectomy decreased by $>30 \%$ between 1998 and 2007. Approximately half of this improvement could be attributed to concentration of these two procedures in higher-volume hospitals. In the Netherlands, minimal volume standards for esophagectomy have markedly reduced the number of hospitals performing that procedure, increased average volumes in remaining centers, and dramatically reduced operative mortality over the past 10 years. ${ }^{5,6}$ In contrast, there have been no coincident trends in either market concentration or mortality with pancreatectomy, for which the Netherlands has not set volume standards. $^{7}$

Why did market concentration in Quebec fail to result in comparable improvements in operative mortality with pancreatic resection? The authors suggest that this finding refutes the importance of provider volumes with this procedure, but this conclusion seems overreaching in light of the considerable literature suggesting otherwise. Instead, findings from this study suggest opportunities for substantial 
quality improvement in the delivery of pancreatic surgery in Quebec. Its most recent mortality rates are considerably higher than population-level benchmarks from the United States, and at least double what would be expected of U.S. hospitals with comparable volumes.

More broadly, findings from the study by Simunovic et al. indicate that laissez-faire market concentration may not be sufficient in improving surgical outcomes. ${ }^{4}$ After all, Cancer Care Ontario established referral standards on the basis of hospitals' mortality rates, not on volume alone. Thus, they avoided the pitfalls associated with redistributing patients to high-volume hospitals with substandard outcomes. Through its active approach to improving quality and regionalization, Cancer Care Ontario no doubt made its provincial hospitals much more aware of their relative performance and may have motivated internal quality improvement activities. Although the specific nature of any such interventions cannot be inferred from this study, the surgical Hawthorne effectthat hospitals tend to improve when their outcomes are being scrutinized-is widely recognized.

The findings from Ontario and new data from other countries suggest that adequate hospital caseloads are important for achieving safe pancreatic resection, but not necessarily sufficient. To ensure acceptable operative mortality rates, high-volume surgeons and hospitals should actively monitor their outcomes and benchmark their performance against their peers. They should also look for opportunities to learn from each other and improve. In the United States, the American College of Surgeons' National Surgical Quality Improvement Program is launching an outcomes tracking program specific to pancreatectomy. ${ }^{8}$ Surgeon leaders from the American Hepato-PancreatoBiliary Association are using this measurement system as a platform for a new national quality improvement collaborative for pancreatic surgery. ${ }^{9}$ Optimizing outcomes with this complex procedure will ultimately require a combination of regionalization, outcomes monitoring, and identification and dissemination of best practices.

\section{REFERENCES}

1. Birkmeyer JD, Siewers AE, Finlayson EV, et al. Hospital volume and surgical mortality in the United States. $N$ Engl $J$ Med. 2002;346:1128-37.

2. Birkmeyer JD, Stukel TA, Siewers AE, et al. Surgeon volume and operative mortality in the United States. $N$ Engl J Med. 2003; 349:2117-27.

3. Milstein A, Galvin RS, Delbanco SF, Salber P, Buck CR Jr. Improving the safety of health care: the leapfrog initiative. Eff Clin Pract. 2000;3:313-6.

4. Simunovic M, Urbach D, Major D, et al. Assessing the volumeoutcome hypothesis and region-level quality improvement interventions: pancreas cancer surgery in two Canadian Provinces. Ann Surg Oncol. 2010. doi:10.1245/s10434-010-1114-0.

5. Wouters MW, Karim-Kos HE, le Cessie S, et al. Centralization of esophageal cancer surgery: does it improve clinical outcome? Ann Surg Oncol. 2009;16:1789-98.

6. Wouters MW, Krijnen P, Le Cessie S, et al. Volume- or outcomebased referral to improve quality of care for esophageal cancer surgery in the Netherlands. J Surg Oncol. 2009;99:481-7.

7. van Heek NT, Kuhlmann KF, Scholten RJ, et al. Hospital volume and mortality after pancreatic resection: a systematic review and an evaluation of intervention in the Netherlands. Ann Surg. 2005;242:781-8.

8. Birkmeyer JD, Shahian DM, Dimick JB, et al. Blueprint for a new American College of Surgeons: National Surgical Quality Improvement Program. J Am Coll Surg. 2008;207:777-82.

9. Pitt HA, Kilbane M, Strasberg SM, et al. ACS-NSQIP has the potential to create an HPB-NSQIP option. HPB (Oxford) 2009;11: 405-13. 